\title{
Evaluation of Risk Area by Myocardial Contrast Echocardiography with a New Computer-aided Method
}

\author{
Jia-Wei Tian ${ }^{1,2}$, Guo-Qing Du ${ }^{1}$, Ying Liu ${ }^{1}$, Min Ren $^{1}$, Ying Wang ${ }^{1}$, Meng \\ Zhang $^{1}$, Xiang-Long Tang ${ }^{2}$, H.D. Cheng ${ }^{3}$, Yan-Hui Guo ${ }^{3}$ \\ ${ }^{1}$ Department of Ultrasound, Second Affiliated Hospital of Harbin Medical University. \\ Harbin 150086. China \\ ${ }^{2}$ School of Computer Science and Technology, Harbin Institute of Technology. Harbin \\ 150001. China \\ ${ }^{3}$ Department of Computer Science, Utah State University. Utah . U.S.A
}

\begin{abstract}
This paper presents a new computeraided method applicable for myocardial contrast echocardiography (MCE) to calibrate myocardial contrast intensity (CI) quantitately and to test the value of color-coded images in evaluating risk area. When the difference of gray level between myocardium and cavity was -70 , the software can identify risk segments and yield the sensitivity of $95 \%$ and specificity $87 \%$. The correlation between the risk area by MPD and fluorescent staining was $0.84(P=0.003)$ whereas color-coded map and staining was 0.91 ( $P$ $<0.001)$. MCE with a new computeraided technique can assess myocardial perfusion quantitatively and identify risk area automatically.
\end{abstract}

Keywords: myocardial contrast echocardiography, computer-aided, myocardial perfusion

This work is supported, in part, by a grant from the Heilongjiang Province Nature and Science Foundation in China (No. ZJY0707-02)

Corresponding Author: Xiang-Long Tang

\section{Introduction}

Myocardial contrast echocardiography (MCE) has been used to gain new insight into diagnosis of myocardial infarct (MI) and/or myocardial ischemia[1-4]. Because contrast agent has similar rheology as red blood cells within the microcirculation, MCE can also assess the myocardial capillary perfusion after thrombolytic therapy or percutaneous coronary intervention[5-7]. The current interpretation of perfusion images by MCE is performed by visual analysis. However, the accuracy of such analysis depends on the investigator's expertise and his/her ability to distinguish artifacts from actual perfusion defects. In addition, it is very difficult to assess the inadequate perfusion only using visual analysis. Quantification of myocardial perfusion with MCE is critical and valuable. Yano et al[8] developed a new calibration technique applicable for MCE in the recognition of infarct size and its spatial extent. They took the ratio of myocardial contrast intensity (CI) to that of $100 \%$ blood pool of the left ventricular (LV) cavity to standardize myocardial CI.

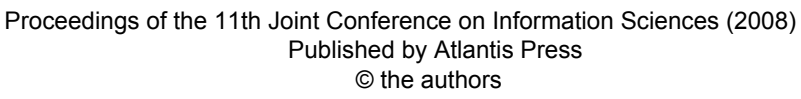


However, these data did not have any validation with an exterenal gold standard. In order to solve these problem, this study presents a new computer-aided method applicable for MCE to average calibrated myocardial CI quantitately and automatically and to test the potential of color-coded images of calibrated CI in assessing myocardial risk area.

\section{Methods}

\subsection{Animal Preparation}

All animal studies were performed in accordance with guidelines for the care and use of laboratory animals of the second affiliated hospital of Harbin Medical University. We anesthetized 30 male rabbits $(2.5 \pm 0.5 \mathrm{~kg})$ by using sodium pentobarbital (30 mg/kg body weight, i.v.). After obtaining baseline images, a left thoracotomy was performed to open the chest, a pericardial cradle was created and the heart was exposed; the left ventricular branch of the left circumflex coronary artery was isolated and occluded with a silk snare, which allowed for later release and reperfusion. After coronary occlusion and reperfusion for different times based on the experimental protocol, the chest was closed in layers.

\subsection{Experimental Protocols}

Two experimental groups were studied. The rabbits underwent 15-min coronary occlusion followed by 30-min reperfusion (15O/30R, Group I, n=15) and 30-min coronary occlusion followed by $60-\mathrm{min}$ reperfusion (300/60R, Group II, $n=15$ ).

At the end of the protocol, all rabbits received heparin (200 U, i.v.) and sodium pentobarbital (50 mg/kg, i.v.). Potassium chloride was then administered intravenously to induce cardiac arrest. The hearts were excised. The ascending aorta was cannulated and perfused (120 mmHg) retrogradely with saline then Zinccadmium sulphide fluorescent microsphere $(0.5 \%)$ after ligation of the previously occluded artery. The hearts were frozen at $-80^{\circ} \mathrm{C}$ overnight hours and then were cut into 8-15 thin slices (5mm) paralleling the coronary sinus toward the apex. Slices were fixed overnight in $10 \%$ formaldehyde and then photographed with use of a digital camera. A computerized planimetric program (Adobe Photoshop, version 7.0) was employed to allowe for quantification of the infarcted zones. The area at risk was identified as the non-fluorescent microsphere region.

\subsection{MCE data Acquisition}

Echocardiographic studies were performed (Vivid 7, GE Medical Systems, USA) by use of a 7S transducer (5-8 $\mathrm{MHz}$ ). The contrast agent used was the commercially available sulphur hexafluoride phospholipid-encapsulated microbubble SonoVue (Bracco Medical Inc., Italy). The ultrasound system was adjusted for low mechanical index (MI $=0.2$ ) before contrast injection, and then SonoVue was injected in a bolus dose of $0.5 \mathrm{ml}$ at a rate of $0.05 \mathrm{ml} / \mathrm{s}$ during occlusion and reperfusion. The MCE images were digitally acquired after peak myocardial opacification until the disappearance of contrast from the myocardium. Contrast-enhanced images were obtained in LV short axis view and digital data of 6-10 consecutive heart cycles were recorded on magneto-optical disks for offline analysis.

\subsection{Analysis of MCE data with a new computer-aided software}

The MCE images of magneto-optical disks were copied to an offline workstation for analysis using software made by ourseleves. First, based on eliminating particle swarm optimization (EPSO) clustering algorithm, the software identify endocardium and epicardium

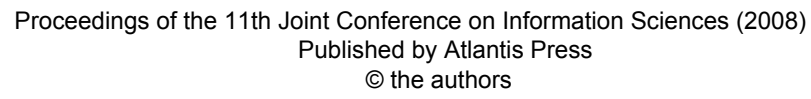


automatically, and then locate the position in myocardium. Second, 6 segments (1=anterior, 2=lateral, 3= poterior, 4=inferior, 5=posterior septum, $6=$ anterior septum) were dissected by the software. Third, the software analyzed each divided segment and each adjacent LV cavity automatically to measure contrast intensity (CI) and obtained the calibrated CI of each myocardial segment by subtracting adjacent cavity CI from myocardial CI[8]. Fourth, a color-coded map of calibrated CI was generated automatically. When the calibrated CI $\leqslant$ 70 , myocardium was colored in red, and those with calibrated $\mathrm{CI}>-70$ were divided into green, blue and yellow by incremental 20. Finally, the end-systolic frame of each segment and its risk areas were defined by MCE as the myocardial perfusional defect (MPD) and color-map during coronary artery occlusion.

\subsection{Statistical analysis}

Data are expressed as means \pm SD. Unpaired Student's $t$-test was used to compare risk segments versus non-risk segments. Comparison of values among different groups were analyzed with oneway ANOVA. Correlation of risk area was calculated between MCE and staining using Kappa scores. Statistical analysis involved SPSS 11.0 for Windows.

\section{Results}

\subsection{General characteristics}

Two rabbits in Group II were excluded because they died during occlusion or reperfusion, so 18 remaining rabbits for analysis. A total of 168 myocardial segments (100\%) were analyzed by staining and 155 segments (92.3\%) by software. 13 segments of MCE images were not analyzed by software because of contrast attenuation, artifacts, or shadowing.

\subsection{Calibrated CI and color-coded map in different groups}

Figure 1 showes values of calibrated CI of myocardial segments in different groups after reperfusion calculated automatically by software. Changes of myocardial CI and calibrated CI in risk and non-risk segments in different groups after reperfusion are described in Table 1. Compared with non-risk segments, myocardial CI values were significantly different in risk segments $(P<0.01)$. However, there was no difference between Groups I and II. Figure 2 displays color-coded map in different groups after reperfusion and there was no red in Groups I and II.

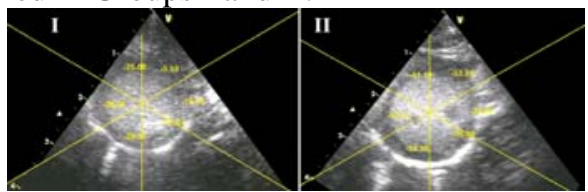

Fig. 1: Calibrated contrast intensity of each segment in different groups calculated automatically by computeraided technique

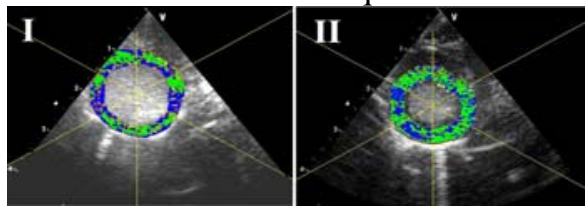

Fig. 2: Color-coded map of calibrated CI in different groups. All segments showed green, blue, and little yellow, implying calibrated CI $>-70$.

\subsection{Correlation of the risk area between MCE and staining}

During coronary occlusion, risk regions in all groups failed to show contrast enhancement were well defined and generally transmural. In contrast, after reperfusion risk regions in Group I and Group II did not exist MPD. Figure 3 shows myocardial contrast, fluorescent

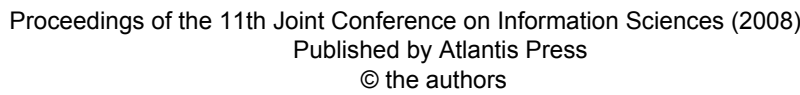


Table 1. Myocardial CI and calibrated CI in risk and non-risk segments in different groups (gray scale, mean \pm SD)

\begin{tabular}{cccc}
\hline & \multirow{2}{*}{ Non-risk } & \multicolumn{2}{c}{ Risk segments } \\
\cline { 3 - 4 } & segments & Group & Group \\
& & I & II \\
\hline Myocardial & $157.02 \pm$ & 142.52 & 136.49 \\
CI & 14.30 & \pm & \pm \\
Calibrated & $-42.46 \pm$ & $-39.75^{*}$ & $19.13^{*}$ \\
CI & 17.03 & \pm 9.56 & -44.07 \\
\hline
\end{tabular}

Group I, 15-min occlusion / 30-min reperfusion; Group II, 30-min occlusion / 60-min reperfusion; CI, contrast intensity. $* P<0.01$ vs. non-risk segments; \# $P<$ 0.01 vs. Group I and Group II.

staining images in different groups. As exhibited, no-fluorescent microsphere regions supplied by left ventricular branch coronary artery were the anatomical risk areas (39.2 $\pm 11.4 \%)$. MCE with MPD and color-map (redcoded) consistently underestimated the risk areas $(28.3 \pm 15.5 \%$ and $32.4 \pm$ $12.7 \%$ ) , which were smaller than that determined by tissue fluorescent staining. When considering the risk area obtained by MCE, correlation with staining was higher when using color-map ( $r=0.91 ; P$ $<0.001)$ than MPD $(r=0.84 ; P=0.003)$.

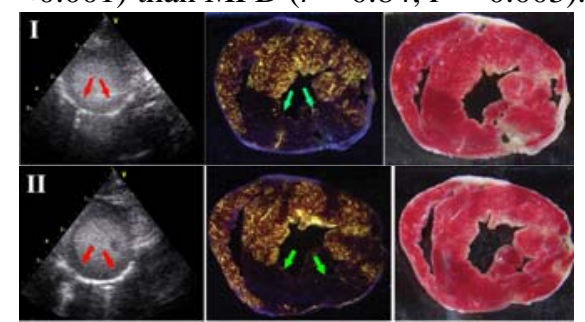

Fig. 3: Comparison of myocardial contrast (left), zinc-cadmium sulphide fluorescent microsphere (middle) and TTC (right) images in different groups. No-fluorescent microsphere regions (middle, green arrow) supplied by left ventricular branch were the anatomical risk areas, which was corresponding to the areas (left, red arrow) in short axis in myocardial contrast echocardiography. Viable myocardium was stained red by TTC (right).

\section{Discussion}

Myocardial contrast echocardiography (MCE) is performed with intravenous administration of encapsulated microbubble contrast agents. These microbubbles remain within the vascular compartment and behave similar to red blood cells in the microcirculation, where they are imaged[9]. Recently, many studies using MCE to assess regional perfusion or viability rely on subjective interpretation of MCE images, which have several limitations[10].

We designed a new quantitative software of MCE based on EPSO clustering algorithm, which can identify endocardium and epicardium automatically, and then locate the position in myocardium. The myocardium in $\mathrm{LV}$ short axis was dissected and analyzed by the software. Average calibrated CI in each segment was calculated and the color-coded map of calibrated CI was drawn, which may represent microvascular perfusion of each segment. Our study results showed that average calibrated CI in each segment was better in differentiating the abnormal perfusion than average myocardial CI and -70 was an appropriate cutoff point to identify risk myocardium. When we analyzed all the rabbits, there was no statistical difference between the risk area predicted by colorcoded map of MCE and that determined by staining. The result shows that MCE with the new computer-aided technique can measure the risk area and necrotic size accurately.

We observed that the risk area determined by MPD was smaller than the anatomical risk area. One possible explanation is that the presence of 
collateral flow from adjacent vascular beds allowing some degree of myocardial perfusion in the margins of regions supplied by the occluded coronary artery [4]. Although the risk area measured by color-coded map of the computer-aided software was also smaller than the anatomical risk area, the correlation between the above measurements was better than that between MPD and staining. For carring out the planimetry of MPD only in the areas with completed absence of myocardial contrast, we did not include regions with hypoperfusion surrounded the central ischemic area in the MPD measurement. On the other hand, the hypoperfusion area was not measured only by visual analysis. In contrast, based on calibrated CI $\leqslant-70$, the color-map not only identified the perfusional defect area, but also signed some degree of hypoperfusion surrounding the defect, which indicates that MCE with color-coded technique was better than MPD in assessing the risk area. In addition, in Groups I and II, MCE with the new technique did not find the infarct size, indicating that short coronary occlusion was not enough to produce myocardial infarction. The results were confirmed by TTC and consistent with previous studies showing that short occlusions in the myocardium ( $<45 \mathrm{~min})$ followed by reperfusion resulted in myocardial stunning $[3,4]$.

Our study shows that MCE with the new computer-aided technique can accurately measure risk area. Using automation methods, this technique can decrease subjectivity of visual evaluation and enhance reproducibility, whereas it may provide a convenient and reliable method at bedside for identification of myocardial infarction. Monitoring the treatment of patients with acute myocardial infarction, the technique can point out the need for more intensive inhospital and follow-up care.

\section{References}

[1] T.R. Porter, F. Xie, A. Kricsfeld and K. Kilzer, "Noninvasive identification of acute myocardial ischemia and reperfusion with contrast ultrasound using intravenous perfluoropropaneexposed sonicated dextrose albumin," J Am Coll Cardiol, vol. 26, pp. 33-40, 1995.

[2] J.M. Swinburn, A. Lahiri and R. Senior, "Intravenous myocardial contrast echocardiography predicts recovery of dysynergic myocardium early after acute myocardial infarction," J Am Coll Cardiol, vol. 38, pp. 19-25, 2001.

[3] S. Lafitte, A. Higashiyama, H. Masugata, B. Peters, M. Strachan, O.L. Kwan and A.N. DeMaria, "Contrast echocardiography can assess risk area and infarct size during coronary occlusion and reperfusion: experimental validation," J Am Coll Cardiol, vol. 39, pp. 1546-1554, 2002.

[4] P.M. Dourado, J.M. Tsutsui, W. Jr. Mathias, J.L. Andrade, P.L. da Luz and A.C. Chagas, "Evaluation of stunned and infarcted canine myocardium by real time myocardial contrast echocardiography,” Braz J Med Biol Res, vol. 36, pp. 1501-1509, 2003.

[5] H. Ito, A. Okamura, K. Iwakura, T. Masuvama, M. Hori, S, Takiuchi, S. Negoro, Y. Nakatsuchi, Y. Taniyama, Y. Higashino, K. Fujii and T. Minamino, "Myocardial perfusion patterns related to thrombolysis in myocardial infarction perfusion grades after coronary angioplasty in patient with acute anterior wall myocardial infarction," Circulation, vol. 93, pp. 1993-1999, 1996.

[6] W. Lepper, R. Hoffmann, O. Kamp, A. Franke, C.C. de Cock, H.P. Kuhl, G.T. Sieswerda, J. Dahl, U. Janssens, P. Voci, C.A. Visser and P. Hanrath, "Assessment of myocardial 
reperfusion by intravenous myocardial contrast echocardiography and coronary flow reserve after primary percutaneous transluminal coronary angioplasty in patients with acute myocardial infarction,” Circulation, vol. 101, pp. 2368-2374, 2000.

[7] M.A. Caldas, J.M. Tsutsui, I. Kowatsch, J.L. Andrade, J.C. Nicolau, J.F. Ramires and W.Jr. Mathias, "Value of myocardial contrast echocardiography for predicting left ventricular remodeling and segmental functional recovery after anterior wall acute myocardial infarction," J Am Soc Echocardiogr, vol. 17, pp. 923932, 2004.

[8] A. Yano, H. Ito, K. Iwakura, R. Kimura, K. Tanaka, A. Okamura, S. Kawano, T. Masuvama and K. Fujii,
"Myocardial contrast echocardiography with a new calibration method can estimate myocardial viability in patients with myocardial infarction,” J Am Coll Cardiol, vol. 43, pp. 1799-1806, 2004.

[9] J.R. Lindner, J. Song, A.R. Jayaweera, J. Sklenar and S. Kaul, "Microvascular rheology of Definity microbubbles following intra-arterial and intravenous administration," J Am Soc Echocardiogr, vol. 15, pp. 396403, 2002.

[10] J.R. Lindner and J. Sklenar, "Placing faith in numbers: quantification of perfusion with myocardial contrast echocardiography,” J Am Coll Cardiol , vol. 43, pp. 1814-1816, 2004. 